\title{
A helicase that gets Oskar's message across
}

\author{
Ilan Davis
}

\begin{abstract}
Messenger RNA localization is a common way of targeting proteins to their site of function. This process is dependent on RNA signals that are interpreted by trans-acting factors. A putative RNA helicase and translational initiation factor is now shown to form a conserved complex, important for localization of oskar mRNA in Drosophila melanogaster and RNA surveillance in human cells.
\end{abstract}

Messenger RNA localization and the translational repression of unlocalized mRNA have a pivotal role in patterning the future embryo within the Drosophila oocyte (Fig. 1). Three key transcripts have been extensively studied: bicoid (bcd), gurken (grk) and oskar (osk). bcd mRNA is localized to the anterior of the oocyte and encodes a morphogen that establishes the anterior-posterior axis in the embryo $^{1}$, whereas grk mRNA is localized near the oocyte nucleus and restricts the distribution of a transforming growth factor $\alpha$ (TGF$\alpha$ ) signal that establishes both the anterior-posterior and dorso-ventral axes in the oocyte. Finally, osk mRNA localization in the posterior, together with the translational repression of unlocalized osk mRNA, targets Osk protein to the posterior. At this site, Osk protein is sufficient to trigger the recruitment of the other mRNAs and proteins that are required to specify the germ cells and abdomen of the future embryo and $\mathrm{fly}^{2}$. All three transcripts require specific microtubuleassociated molecular motors for their localization $^{3-5}$, and a key remaining question is how RNA signals are interpreted by trans-acting factors and sorted to their correct destinations by molecular motors. Reporting in Nature $^{6}$, Palacios et al. now identify the putative RNA helicase and translational initiation factor eIF4AIII as a new factor required for osk mRNA localization. They suggest that specificity may be conferred by the interactions of multiple factors at different stages of an mRNA's transit.

A priori, it seemed reasonable to assume that the sorting of transcripts to distinct destinations would be defined by a simple RNA signal to which a single trans-acting factor binds, providing a specific link to an appropriate motor. Indeed, this is the case for yeast ASH1 mRNA: She2p and She3p link ASH1 RNA to the myosin motor required for its localization ${ }^{7}$. However, it is not that simple in Drosophila. Single-specificity determinants

Ilan Davis is in the Wellcome Trust Centre for Cell Biology, University of Edinburgh, Mayfield Road, Edinburgh EH9 3JR, UK.

e-mail:ilan.davis@ed.ac.uk

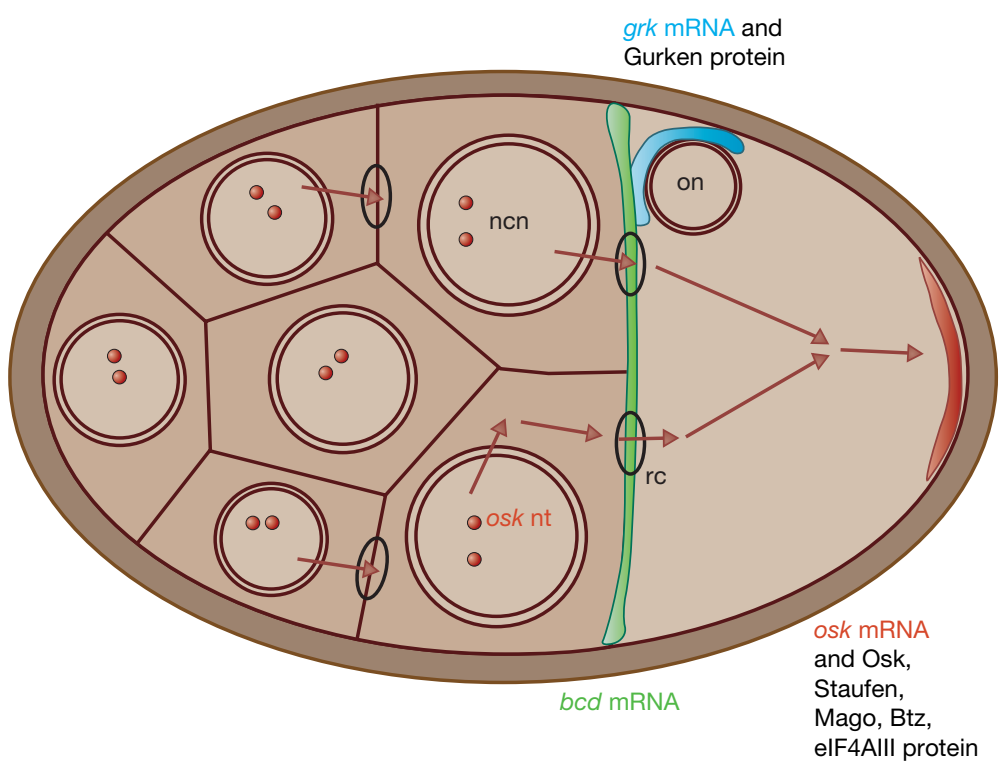

Figure 1 Ribonucleoprotein sorting during fly oogenesis. A schematic representation of a Drosophila melanogaster egg chamber, showing the localization of three well-characterized transcripts, bicoid (bcd; green), gurken (grk; blue) and oskar (osk; red), which together pattern the oocyte and future embryo, and specify the germ line. osk mRNA is transcribed (represented as nascent transcripts (osk nt)) in the nurse cell nuclei (ncn) and transported through actin-rich ring canals $(r c)$ into the oocyte, where it localizes to the posterior pole. osk mRNA localization, coupled with translational repression of unlocalized osk mRNA, targets the Osk protein to the posterior and results in recruitment of many posterior pole components, including Vasa and Nanos. grk mRNA is localized in a cap near the oocyte nucleus (on), whereas bcd mRNA is localized at the anterior of the oocyte. Palacios et al. now show that elFAIII also localizes to the posterior pole together with Btz and Mago and is important for osk mRNA localization.

have not been found for $b c d$, osk or grk mRNA, and specific destinations are most probably defined by many trans-acting factors that vary considerably in their composition during different stages of the complex journey of an RNA - from its site of transcription to its final cytoplasmic destination. For example, the bcd 3'-untranslated region (UTR) localization signal is decorated by several factors that might all be involved in its localization ${ }^{8}$. Similarly, osk mRNA always colocalizes with Staufen (a double-stranded RNA-binding protein essential for its localization), but Staufen alone cannot determine its transcript destination as it also binds to $b c d$. Furthermore, osk mRNA localization requires, among other factors, a nuclear exon junction complex (EJC) ${ }^{9}$ containing Mago nashi (Mago) and Y14 (Mago-Y14), which has been shown in human cells to mark the location on a spliced transcript where introns have been removed.

Palacios and colleagues ${ }^{6}$ add an exciting new layer of complexity to how cytoplasmic transcript destination is selected, at least in the case of osk mRNA. They show that Barentsz (Btz) - a trans-acting factor required for osk mRNA localization - interacts with Drosophila eIF4AIII both genetically and physically. EIF4AIII is a DEAD-box helicase that is homologous to the translation initiation factor eIF4AI, although its role in translation remains unclear $^{10}$. So is eIF4AIII required, similarly to Btz, for osk mRNA localization, 


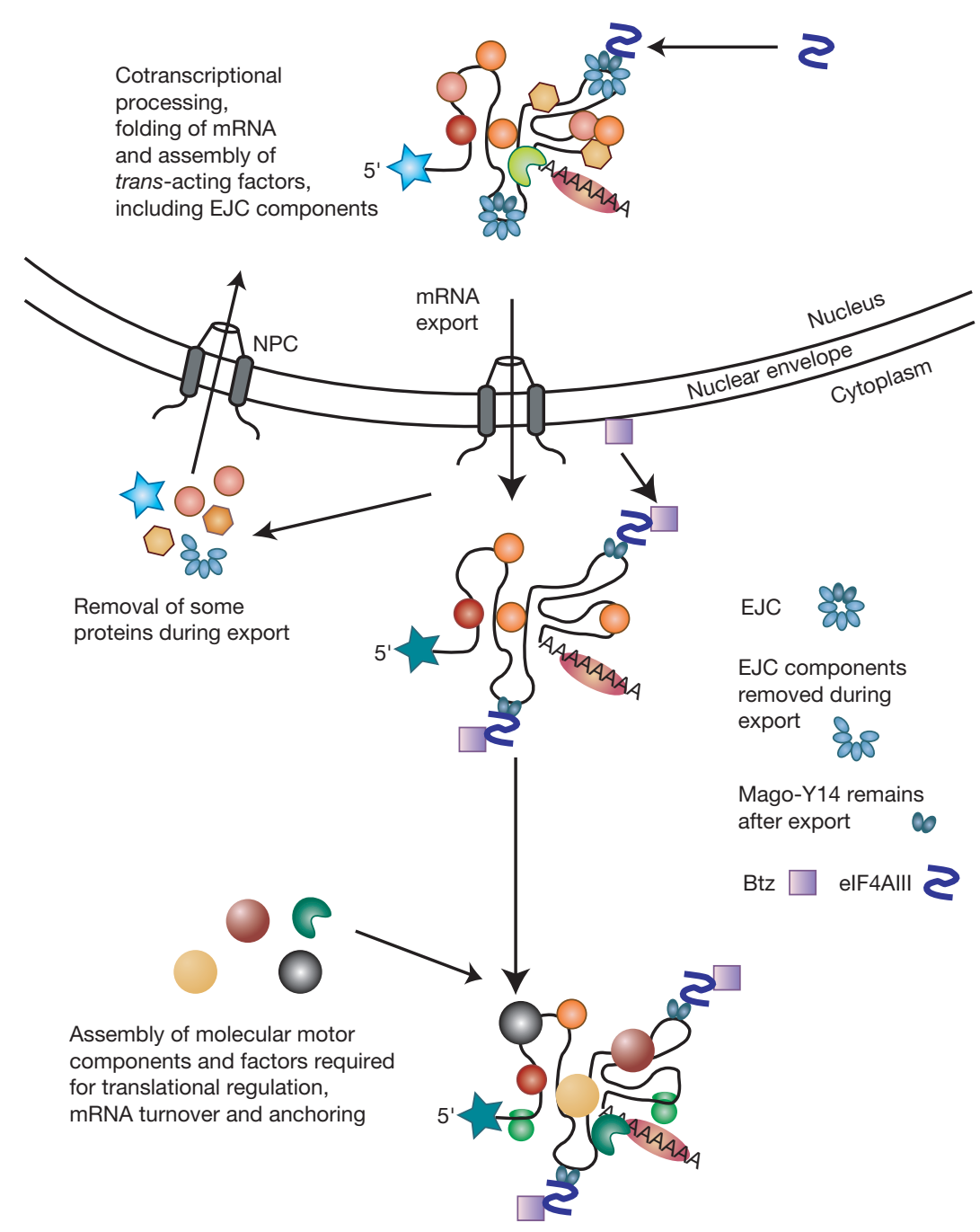

Figure 2 elF4AIII, Btz and Mago-Y14 may be recruited at distinct stages. Many proteins assemble on an RNA cotranscriptionally, such as factors required for processing (splicing and polyadenylation), cap-binding proteins and the exon junction complex (which marks the site of intron removal). After release of the mature transcript, it arrives at the nuclear pore complex (NPC) for export to the cytoplasm. During export, many proteins are removed from the RNP, others attach during export, whereas others remain attached throughout the process. Many of the proteins that are removed are shuttling proteins that are re-imported into the nucleus, and some are required for the export process itself. In the cytoplasm, many new protein complexes are assembled, including factors required during transport by molecular motors, anchoring, translational regulation and mRNA turnover. Palacios et al. now propose that osk mRNA recruits elF4AIII in the nucleus together with components of the EJC, such as Mago-Y14, and then elF4III helps recruit Btz on the cytoplasmic face of the nuclear envelope, while the osk RNP is being exported and remodelled.

and is eIF4AIII localized to the posterior pole in a similar way to Btz, Mago-Y14, and Staufen? Unfortunately, the only available eIF4AIII mutation is homozygous lethal, and germ-line clones (containing homozygous egg chambers in heterozygous viable mothers) are too sick to analyse in detail. This is presumably because the protein is required for correct expression of many RNAs in the cell. However, Palacios et al. were able to show that a heterozygous eIF4AIII mutation, which is otherwise normal, strongly enhances the osk mRNA mis-localization phenotype of a $b t z$ hypomorphic mutant oocyte, as well as disrupting localization of the Staufen protein. Similarly, eIF4AIII-heterozygous embryos show strong abdominal defects in the appropriate $b t z$ mutant background. So, clearly eIF4AIII is important for both osk mRNA localization and abdomen formation. Consistently, the authors show that eIF4AIII protein is colocalized with Mago at the posterior pole and shows a Mago-Y14like nuclear distribution in all cells in the egg chamber, including the nurse cell nuclei, where osk mRNA originates. In addition, Palacios et al. demonstrate that eIF4AIII also exists in a complex with Mago-Y14 and Btz in human cells.

Components of the EJC are required for nonsense-mediated decay (NMD) in human cells ${ }^{11}$, although they have been shown to be dispensable in Drosophila S2 cells ${ }^{12}$. This process - important for preventing the cytotoxic effects of truncated proteins - promotes the identification and rapid degradation of messages containing premature stop codons. Palacios et al. used RNA interference in human cells to test the possible role of Btz and eIF4AIII in NMD, using two established reporters. They found that depletion of Btz and eIF4AIII reduced NMD to a similar extent as Mago-Y14. The authors then used an elegant 'NMD tethering' assay, in which they artificially linked a factor to a tagged message and tested the factor's ability to induce NMD. They found that Y14 and Btz are each sufficient to induce the NMD machinery, whereas eIF4AIII is not, perhaps because the fusion of eIF4AIII to mRNA destroys its putative helicase activity. However, it is unclear why tethered eIF4AIII would not be able to recruit Btz and then recruit the NMD machinery indirectly. In any case, what is clear is that this complex is important for NMD in humans.

An interesting observation made by the authors is that Btz and eIF4AIII seem to localize to distinct sub-cellular compartments. In Drosophila egg chambers and human cells, eIF4AIII and Mago-Y14 are predominantly nuclear, whereas Btz is cytoplasmic. Extrapolating from this, the authors hypothesize that the assembly of osk ribonucleoproteins (mRNPs) occurs in a stepwise manner. First, Mago-Y14 and eIF4AIII bind osk mRNA in the nucleus. Then Btz is recruited on the cytoplasmic face of the nuclear envelope, through eIF4AIII, as the complex is being exported to the cytoplasm. This idea is indeed attractive, as it is clear from studying the composition of RNPs before and after nuclear export that many proteins are either removed or added onto mRNA during export, whereas others are remodelled on the nuclear envelope. After export, RNA helicases are probably required for RNP remodelling in the cytoplasm ${ }^{12,13}$ (Fig. 2). It also seems probable that the composition of the osk RNP would be complex and ever changing with each step of its journey: from its site of transcription within nurse cell nuclei, through its movement between the nurse cells and oocyte, to its multistep localization within 
the oocyte cytoplasm. Conclusive determination of which proteins are bound to osk mRNA at each step of this complex journey will require new approaches. Biochemical extracts cannot detect subtle changes in the components that are bound to an mRNA at distinct steps of its localization, and immunolocalization or green fluorescent protein (GFP) fusion data cannot exclude the possibility that a protein present at levels below the detection threshold is required. Investigating the subtle changes in RNP composition within living cells is likely to require the ability to detect rapidly changing physical interactions between single protein and mRNA molecules using new and emerging imaging technologies ${ }^{14}$.

Nonetheless, Palacios et al. have shown that not only are nuclear EJC components required for osk localization in the cytoplasm, but also that a putative RNA helicase and translational regulation factor that functions in NMD in humans is also required for osk mRNA localization. This raises many exciting questions. For example, is the RNA helicase activity of eIF4AIII required to remodel RNP composition? Could eIF4AIII be involved in translational inhibition of osk mRNA while it is being transported to the posterior cytoplasm? Is eIF4AIII required for the localization of other transcripts in flies and human neurons? Whatever the answers to these questions, it is probable that even more trans-acting factors with diverse roles in RNA biogenesis will be required for osk mRNA localization and it is unlikely that the specificity of osk mRNA sorting in the cytoplasm could be provided by a single factor. Instead, mRNA localization in the oocyte is likely to be just as complex as mRNA processing and export, or most other RNA-based processes $^{12,13}$. In the final analysis, cytoplasmic destination may be selected by many factors binding RNA indiscriminately with specificity being provided by the overall geometry of a complex.

1. Ephrussi, A. \& St Johnston, D. Cell 116, 143-152 (2004).

2. Ephrussi, A. \& Lehmann, R. Nature 358, 387-392 (1992).

3. Tekotte, H. \& Davis, I. Trends Genet. 18, 636-642 (2002).

4. MacDougall, N., Clark, A., MacDougall, E. \& Davis, I. Dev. Cell 4, 307-319 (2003).

5. Brendza, R. P., Serbus, L. R., Duffy, J. B. \& Saxton, W. M. Science 289, 2120-2122 (2000).

6. Palacios, I. M., Gatfield, D., St Johnston, D. \& Izaurralde, E. Nature 427, 753-757 (2004).

7. Jansen, R. P. Nature Rev. Mol. Cell Biol. 2, 247-256 (2001).

8. Arn, E. A., Cha, B. J., Theurkauf, W. E. \& Macdonald, P. M. Dev. Cell 4, 41-51 (2003).

9. Palacios, I. M. Curr. Biol. 12, R50-R52 (2002).

10. Chan, C. C. et al. RNA 10, 200-209 (2004).

11. Gatfield, D., Unterholzner, L., Ciccarelli, F. D., Bork, P. \& Izaurralde, E. EMBO J. 22, 3960-3970 (2003).

12. Stutz, F. \& Izaurralde, E. Trends Cell Biol. 13, 319-327 (2003).

13. Mitchell, P. \& Tollervey, D. Curr. Opin. Cell Biol. 13, 320-325 (2001).

14. Tsien, R. Y. Nature Rev. Mol. Cell Biol. 4, Suppl, SS16-SS21 (2003).

\section{New neurons?}

The identification and characterization of neural stem cells within different regions of the adult brain, particularly in rodents, has revolutionized our understanding of adult neurogenesis and heightened hopes for treatments of traumatic damage and neurodegenerative disease. However, the degree to which this occurs in the adult human central nervous system has been less well characterized. Recently, Avlarez-Buylla and colleagues (Nature 427, 740-744 (2004)) identified some similarities, as well as some striking species-specific differences, for a pool of neural stem cells that reside in a specialized region of the human sub-ventricular zone (SVZ) - a part of the forebrain that lines the ventricular surface.

In mammals, the adult brain has two main areas that produce precursors for the generation of new neurons: the SVZ and the dentate gyrus of the hippocampus. In rodents, the SVZ produces a train of chain-migrating, newly formed neurons - the rostral migratory stream - that replace a population of inter-neurons in the olfactory bulb. Although human brains were known to contain neural precursors, the precise anatomical localization of these precursors and the degree to which they produce new neurons (particularly outside the dentate gyrus) remained relatively unclear.

Now, Alvarez-Buylla and colleagues use large numbers of cells from human biopsy and autopsy tissue samples to characterize the human neural stem cell pool. They find that the stem cells are localized to a ribbon-like layer of astrocytes lining the inner SVZ; this pronounced layer of the SVZ has not been identified in other mammalian species investigated. This population of astrocytes contained a small proportion $(\sim 4 \%)$ of cells that could be clonally expanded to produce both neuronal and glial cell lineages. Thus, it is clear that the SVZ in humans contains potential neural stem cells. However, the authors find no strong evidence to

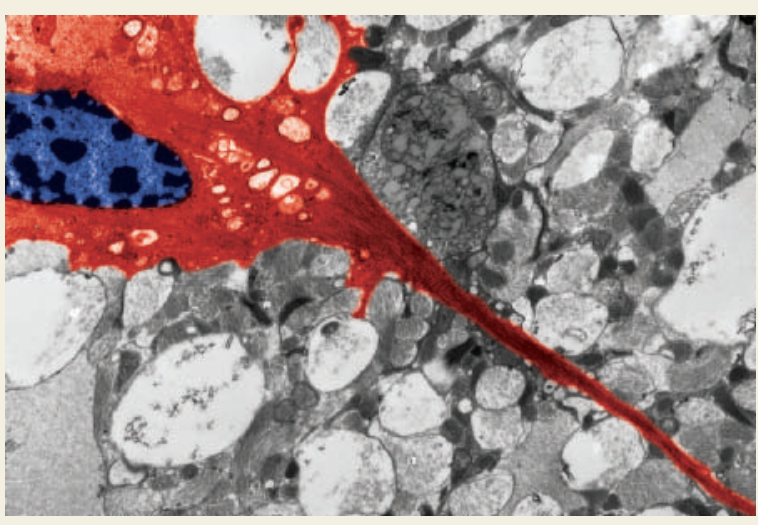

An electron micrograph of single astrocyte (blue nucleus) within the adult human SVZ astrocyte ribbon, which is extending a process of intermediate filaments (red) through the 'gap' region towards the ependyma.

suggest that that these cells are producing differentiated neurons in vivo. Most strikingly, they seem to completely lack the rostral migratory stream that is so prominent in rodents and that has even been identified in primate species.

An important conclusion that can be drawn from this work is that although humans clearly possess neural precursor cells that could potentially be used to replace areas of damaged neurons, the situation in adult humans is clearly different from that in other mammalian species. These differences have obvious implications for interpreting animal studies as a basis for human therapies. Uncovering the molecular underpinning of these differences will be necessary for deriving successful cell-replacement treatments for traumatic and neurodegenerative brain disorders.

JACK HORNE 